CLINICAL STUDY

\title{
GH responses to two consecutive bouts of neuromuscular electrical stimulation in healthy adults
}

\author{
A Sartorio ${ }^{1,2}, \mathrm{M} \mathrm{Jubeau}^{3}, \mathrm{~F}_{\text {Agosti }}{ }^{2},{\mathrm{~A} \mathrm{De} \mathrm{Col}^{2}, \mathrm{~N} \mathrm{Marazzi}^{2}, \mathrm{C} \text { L Lafortuna }}^{4}$ and N A Maffiuletti ${ }^{3,5}$ \\ ${ }^{1}$ Istituto Auxologico Italiano, IRCCS, Divisione Malattie Metaboliche 3 and ${ }^{2}$ Laboratorio Sperimentale di Ricerche Auxo-Endocrinologiche, 28921 \\ Verbania, Italy, ${ }^{3}$ Laboratory INSERM U887 Motricity-Plasticity, Faculty of Sports Sciences, University of Burgundy, 21078 Dijon, France, ${ }^{4}$ Institute of \\ Bioimaging and Molecular Physiology, CNR, 20090 Milan, Italy and ${ }^{5}$ Neuromuscular Research Laboratory, Schulthess Clinic, 8008 Zurich, Switzerland \\ (Correspondence should be addressed to A Sartorio who is now at Laboratorio Sperimentale di Ricerche Auxo-Endocrinologiche, Istituto Auxologico Italiano, \\ IRCCS, Via Ariosto 13, 20145 Milan, Italy; Email: sartorio@auxologico.it)
}

\begin{abstract}
Background: It is well established that repeated GHRH administration or repeated voluntary exercise bouts are associated with a complete blunting of GH responsiveness when the administration of the second stimulus follows the first one after a 2-h interval.

Aim: To evaluate GH responses to neuromuscular electrical stimulation (NMES) in healthy adults. Methods: Six volunteers (mean age \pm s.D. $31.7 \pm 5.5$ years) were studied before and after two consecutive bouts of NMES exercise (a series of 20 contractions at the maximum of individual tolerance, frequency: $75 \mathrm{~Hz}$, pulse duration: $400 \mu \mathrm{s}$, on-off ratio: 6.25-20 s) administered at a 2-h interval.

Results: Baseline GH levels (mean: $0.3 \pm 0.2 \mathrm{ng} / \mathrm{ml}$ ) significantly increased after the first NMES (peak: $4.2 \pm 3.7 \mathrm{ng} / \mathrm{ml})$, with a complete normalization after $120 \mathrm{~min}(0.3 \pm 0.3 \mathrm{ng} / \mathrm{ml})$. The administration of the second bout of NMES of comparable characteristics also resulted in a significant GH increase (peak: $5.2 \pm 3.2 \mathrm{ng} / \mathrm{ml}$ ), which was comparable with that observed after the previous one. GH net incremental area under the curve after the first and second bouts of NMES were not significantly different $(155.1 \pm 148.5$ and $176.9 \pm 123.3 \mathrm{ng} / \mathrm{ml}$ per $\mathrm{h}, \mathrm{P}=0.785)$.

Conclusions: Unlike repeated pharmacological stimuli and voluntary exercise bouts, subsequent sessions of NMES administered at a 2-h interval appear to circumvent feedback mechanisms and to re-induce the $\mathrm{GH}$ responses, thus indicating a possible different underlying mechanism elicited by different GH-releasing stimuli.
\end{abstract}

European Journal of Endocrinology 158 311-316

\section{Introduction}

The secretion of growth hormone $(\mathrm{GH})$ is under the influence of hypothalamic GH-releasing hormone (GHRH) and somatostatin. GH regulates its own secretion via a negative auto-feedback mechanism, which might operate at both the pituitary and hypothalamic levels (1). Increased GH concentrations evoke prompt hypothalamic somatostatin release, which in turn inhibits GHRH secretion, blocks pituitary exocytosis of $\mathrm{GH}$ and sensitizes somatotrophs to the next GHRH stimulus (1).

It is well known that repeated GHRH stimuli administered at 2-h intervals are associated with a complete blunting of $\mathrm{GH}$ responsiveness to the second stimulus in healthy adults $(2-5)$, with a restoration of GH responsiveness occurring when the time elapsed between the two pharmacological stimuli was more prolonged.

Similar results have been reported with repeated bouts of voluntary exercise, an attenuation of GH response being observed if the bouts of high-intensity exercise are repeated within $1-2 \mathrm{~h}(6-8)$. In a similar way to the pattern recorded with repeated GHRH administration, normal GH responsiveness reappeared progressively as the time interval increased between the two consecutive stimuli. When the interval between the two stimuli was $6 \mathrm{~h}$, the GH response was comparable with that observed after the first exercise bout (8).

Neuromuscular electrical stimulation (NMES) is the application of electrical stimulation to produce skeletal muscular contractions as a result of the percutaneous stimulation of peripheral nerves (9). In the last few years, NMES has been used in rehabilitation, geriatric and sport medicine (for review, $(9,10)$ ), with the general objectives to a) minimize the effects of prolonged immobilization or disuse on skeletal muscle structure and function and b) improve neuromuscular function parameters in healthy individuals. More recently, the acute effects of NMES on neuromuscular, metabolic and hormonal responses have also received attention (11-13). We have demonstrated that a single bout of NMES exercise is able to show GH responsiveness in healthy subjects (13). However, GH response after 
a series of 40 stimulated contractions was lower than that observed after pharmacological and/or voluntary exercise stimuli $(4,8)$. It could therefore be hypothesized that repeated bouts of NMES exercise, separated by $2 \mathrm{~h}$, did not induce an attenuation of GH release, that had been observed during repeated maximal cycle exercise (7), due to the low GH response in the first bout.

The aim of the present study was to evaluate changes in $\mathrm{GH}$ and cortisol concentration, blood lactate and muscle strength after repeated bouts of NMES exercise in a group of healthy adults.

\section{Materials and methods}

\section{Subjects}

Six healthy male adults, recruited from friends and colleagues (mean age \pm s.D.: $31.7 \pm 5.5$ years, range: 25-41 years; mean weight: $76.7 \pm 9.2 \mathrm{~kg}$, range: 64.9-88.7 kg; mean body mass index (BMI): $23.6 \pm$ $2.5 \mathrm{~kg} / \mathrm{m}^{2}$, range: $21.2-26.8 \mathrm{~kg} / \mathrm{m}^{2}$ ), volunteered to participate in this investigation after giving their written informed consent. The study protocol was approved by the Ethical Committee of the Italian Institute for Auxology. All the subjects were habitually active but were not involved in strength or endurance training on a regular basis, and none of them had any signs of musculoskeletal disorders. The subjects were asked not to perform any strenuous exercise for at least $48 \mathrm{~h}$ prior to the experiment. None of the subjects reported having ingested drugs or nutritional supplements known to interfere with $\mathrm{GH}$ and cortisol secretion.

The subjects reported to the laboratory $1 \mathrm{~h}$ before the start of the tests, after an overnight fast (10-14 h); all tests started between 0800 and 0830 h. Subjects were positioned in a semirecumbent position. An i.v. cannula for blood sampling was inserted into an antecubital vein, kept patent with isotonic saline and maintained in position for the whole duration of the experiment.

After a standardized warm-up protocol, consisting of submaximal NMES (duration: $5 \mathrm{~min}$, frequency: $5 \mathrm{~Hz}$, pulse duration: $350 \mu \mathrm{s}$ ), the subjects performed two exercise bouts of NMES separated by $2 \mathrm{~h}$ and exerciseinduced changes in muscle strength, GH, cortisol and blood lactate were compared between the two exercise bouts. Additionally, maximal voluntary contraction (MVC) force measurements were performed before and immediately after each bout of NMES exercise to quantify the level of muscle fatigue. The general flow chart of the experimental protocol is shown in Fig. 1.

\section{Exercise}

The quadriceps femoris muscles of both legs were exercised simultaneously using standard surface NMES techniques (13). Two bouts of 20 isometric NMES contractions (9-min duration) were administered separated by a $2-\mathrm{h}$ interval. During exercise bouts, subjects were in supine position on a horizontal leg press machine (Technogym, Gambettola, Italy), with the trunk-thigh and thigh-shank angles at $80^{\circ}$. The leg press force output was measured by a strain gauge (Globus Italia, Codognè, Italy) correctly mounted on the leg press machine, and the signal from the strain gauge was sampled at $100 \mathrm{~Hz}$ and stored on a computer for later analysis with commercially available software (TCS-SUITE 400; Globus Italia).

\section{NMES}

A portable electrical stimulator (Compex Sport-P; Medicompex SA, Ecublens, Switzerland) was used to deliver biphasic symmetric rectangular pulses with the following characteristics: frequency, $75 \mathrm{~Hz}$; pulse duration, $400 \mu \mathrm{s}$; on-off ratio, 6.25-20 s (rise time: $1.5 \mathrm{~s}$ and fall time: $0.75 \mathrm{~s}$ ). Each contraction lasted

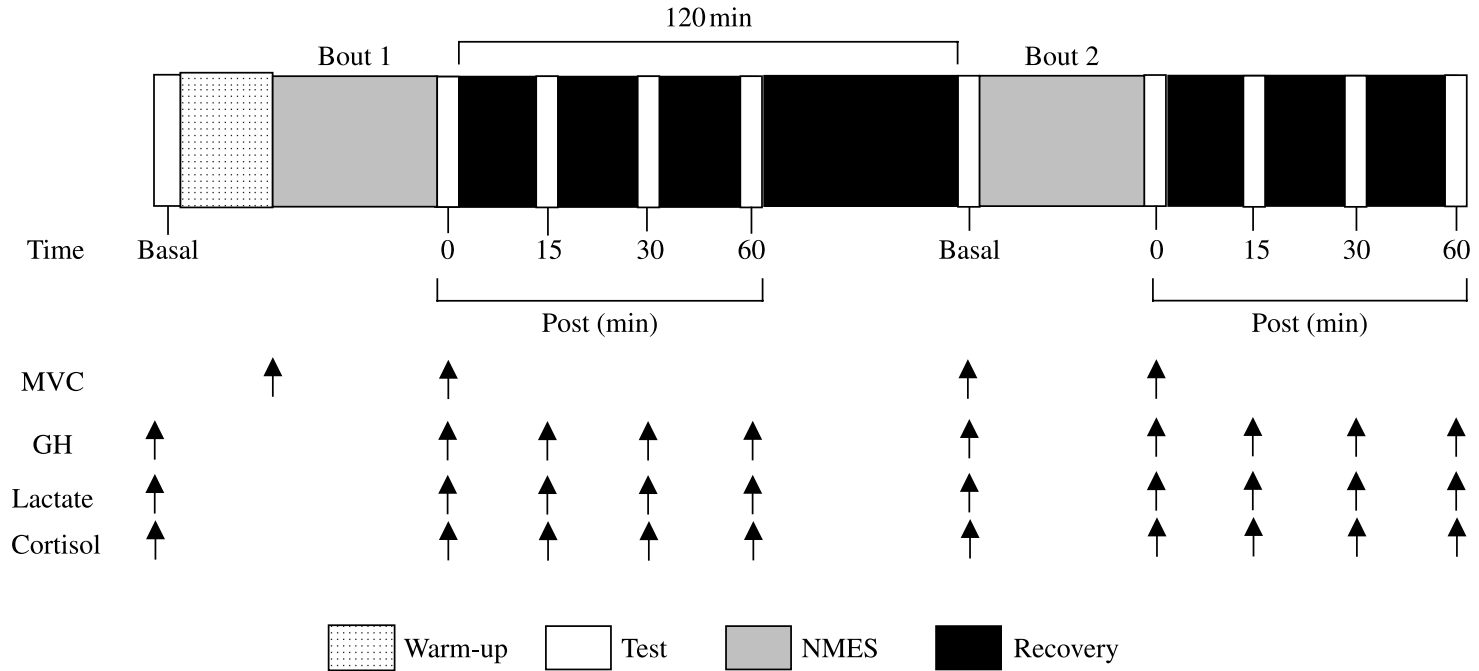

Figure 1 General flow chart of the experimental protocol. Maximal voluntary contraction, MVC; neuromuscular electrical stimulation (NMES). 
$6.25 \mathrm{~s}$ (rise time: $1.5 \mathrm{~s}$ and fall time: $0.75 \mathrm{~s}$ ) followed by a 20-s rest period. Three adhesive electrodes (thickness $2 \mathrm{~mm}$ ) were placed over the anterior part of each thigh. The two positive electrodes $(5 \times 5 \mathrm{~cm})$ were positioned over the motor point of the vastus lateralis and vastus medialis muscles. The negative electrode $(10 \times 5 \mathrm{~cm})$ was placed close to the proximal insertion of the quadriceps femoris muscle $(\sim 5 \mathrm{~cm}$ below the inguinal ligament). The current amplitude (range: 33-110 mA) was consistently increased throughout the 20 electrically evoked contractions to the maximal tolerable level (pain threshold). Identical current amplitudes were used for the right and left quadriceps. The average level of isometric force evoked during each bout of NMES was $26 \pm 8 \%$ (first bout) and $26 \pm 9 \%$ (second bout) of the MVC force recorded before each bout.

\section{MVC}

Before and after the two NMES bouts, subjects were asked to perform bilateral isometric MVC using the leg press machine described previously. The duration of each contraction was $5 \mathrm{~s}$, and the measurement was taken three times with $80 \mathrm{~s}$ of rest between contractions. The highest MVC force value of the three measurements was used for further analysis.

\section{Blood sampling and measurements}

Venous blood samples $(5 \mathrm{ml})$ were taken at the beginning of each NMES bout (i.e. basal values; Fig. 1) and at 15-min intervals thereafter up to $60 \mathrm{~min}$ after the end of each NMES session. Blood samples were allowed to clot, centrifuged and immediately stored at $-20{ }^{\circ} \mathrm{C}$ for the subsequent analyses.

The GH and cortisol concentrations were determined using commercial immunometric kits (Immulite 2000; DPC, Los Angeles, CA, USA); intra- and inter-assay coefficients of variation were 2.5 and $6 \%$ for $\mathrm{GH}$ and 6.5 and $9.6 \%$ for cortisol respectively.

Lactate concentrations were determined using a blood drop taken from the ear lobe (Arkray Inc., Kyoto, Japan); intra- and inter-assay coefficients of variation were 3 and $7 \%$ respectively.

All the samples were run in the same session to minimize inter-assay variability. Volunteers were allowed to drink moderate amounts of water between the two bouts of exercise.

Integrated $\mathrm{GH}$ concentrations (net area under the curve, nAUC) for 60-min time periods (from the end of NMES up to $60 \mathrm{~min}$ after) were calculated using the trapezoidal method (14).

\section{Statistical analysis}

Differences in GH nAUC between the first and second bouts were evaluated with Student's t-test, as appropriate.
The difference in the response of GH, cortisol lactate and MVC to the first and second bouts of NMES at different times after stimulation were evaluated by means of a two-factor ANOVA with repeated measures. When a significant main effect (bout or time) or interaction (bout $\times$ time) was found, a least significant difference (LSD) post hoc test was performed. The statistical significance was set at $P<0.05$.

\section{Results}

Mean GH responses to the two consecutive NMES bouts are shown in Fig. 2A. Mean baseline GH levels were comparable $(P=0.89)$ between the first and second bouts.

The ANOVA applied to NMES-induced GH responses revealed significant changes in $\mathrm{GH}$ at different times, without significant differences between the first and second bouts $(P=0.964)$. A significant bout $\times$ time interaction was also observed. In fact, $\mathrm{GH}$ peaks were comparable and significantly different from the respective basal values, but occurred at different times for the two NMES bouts (at 15 and $30 \mathrm{~min}$ after the end of the first and second bouts respectively; Fig. 2A). In the same way, GH nAUC after the first $(155.1 \pm 148.5 \mathrm{ng} / \mathrm{ml}$ per $\mathrm{h})$ and second NMES bouts $(176.9 \pm 123.3 \mathrm{ng} / \mathrm{ml}$ per $\mathrm{h})$ were comparable $(P=0.785)$.

The MVC force decreased significantly immediately after the first $(-22 \%)$ and second $(-17 \%)$ bouts (total MVC decrease: $-37 \%$ ), but the reduction was not significantly different between the two bouts $(P=0.204)$. However, the MVC force before the second bout $(2073 \pm 261 \mathrm{~N})$ was significantly lower than that before the first bout $(2708 \pm 282 \mathrm{~N})$.

Mean blood lactate levels are reported in Fig. 2B. Mean baseline lactate concentrations were comparable $(P=0.103)$ between the first and second bouts. The ANOVA applied to NMES-induced lactate responses revealed significant changes in lactate at different times, without significant differences between the first and second bouts $(P=0.172)$ and without bout $\times$ time interaction $(P=0.442)$. Blood lactate significantly increased immediately after the first and second NMES bouts in a similar way (Fig. 2B).

Mean cortisol levels are reported in Fig. 2C. Mean baseline cortisol concentrations were significantly different between the first $(14.2 \pm 3.9 \mathrm{ng} / \mathrm{ml})$ and second bouts $(8.4 \pm 1.2 \mathrm{ng} / \mathrm{ml})$.

The ANOVA applied to NMES-induced cortisol responses revealed a significant bout $\times$ time interaction. As shown in Fig. 2C, a significant decrease in the cortisol level was observed at the end of the first bout (post $60 \mathrm{~min}$ ), while the second bout produced a significant increase in cortisol immediately after the stimulation. This increase remained significantly elevated and peaked $15 \mathrm{~min}$ after NMES. However, it is interesting to note that cortisol concentrations measured immediately after, as well as $30 \mathrm{~min}$ after, the second NMES bout were 
A

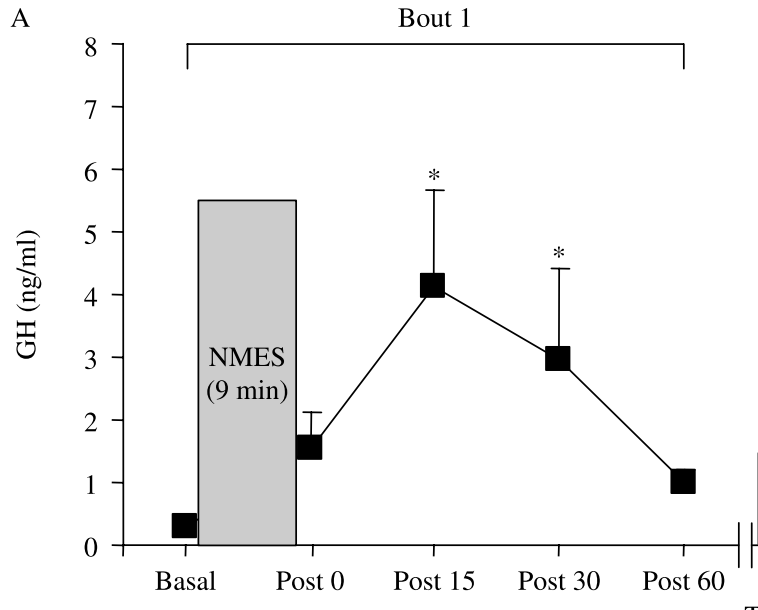

B

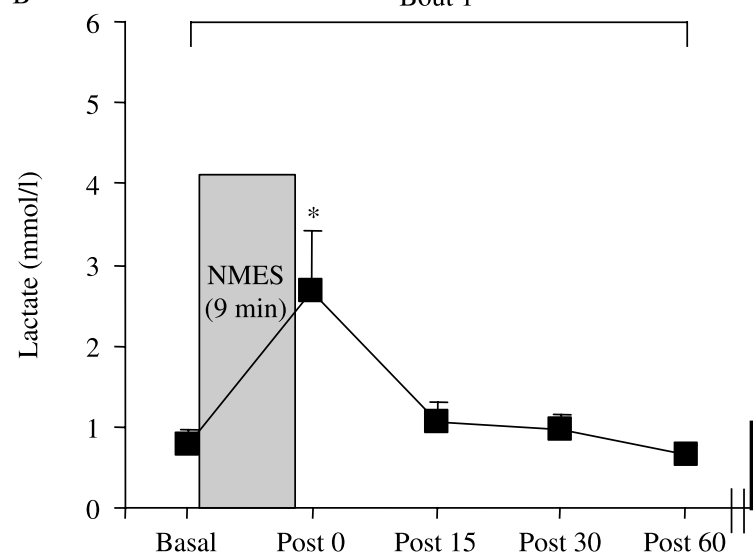

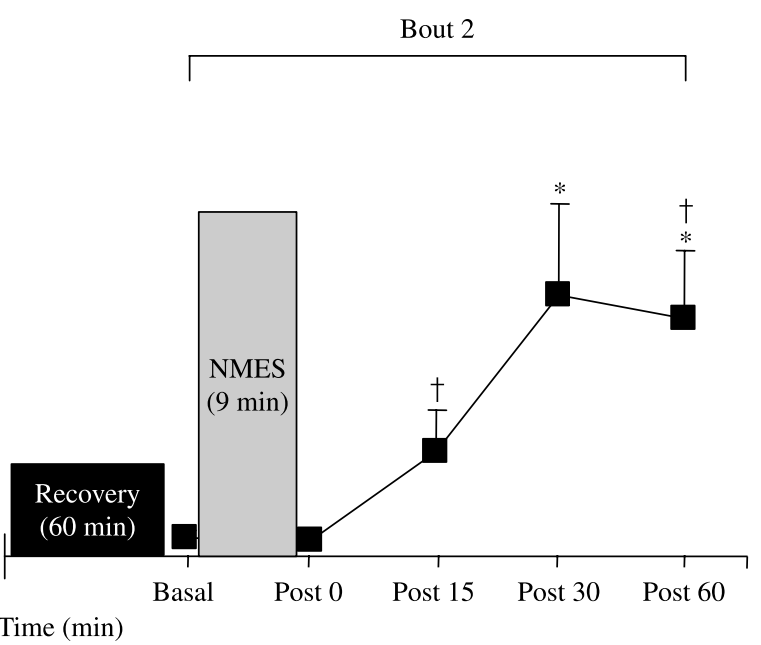

Time (min)
Bout 2

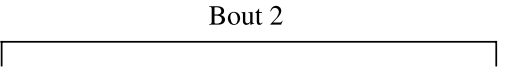

Recovery $(60 \mathrm{~min})$

Time (min)

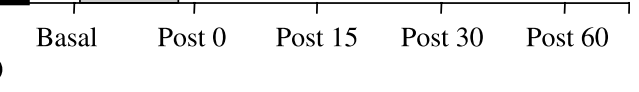

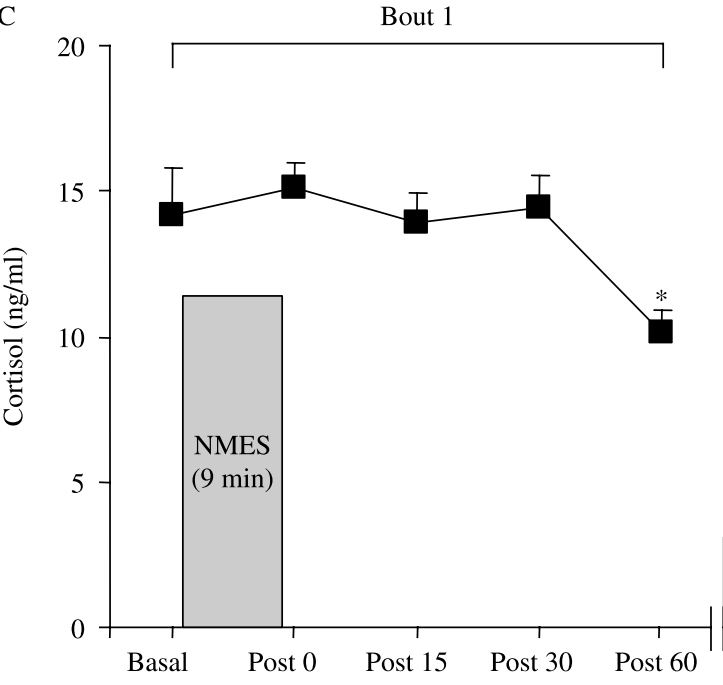

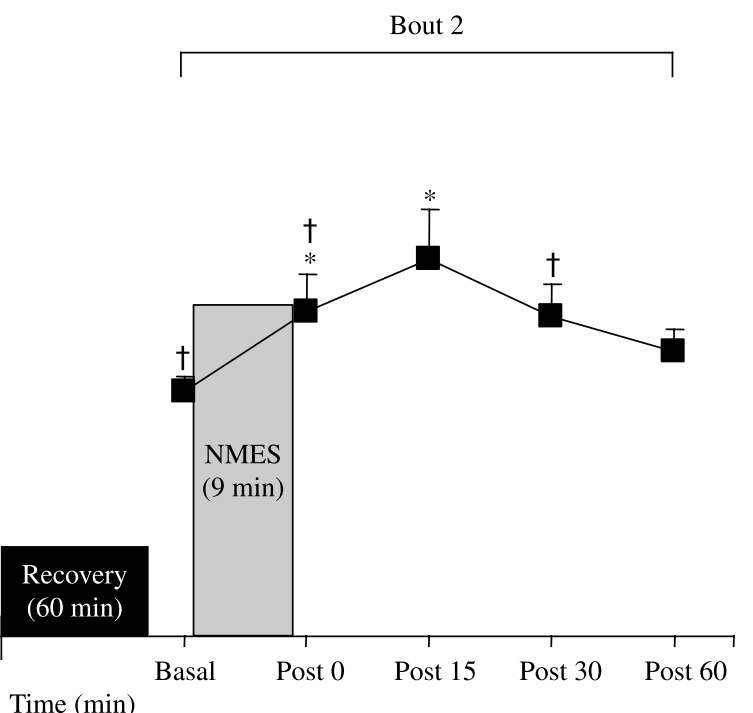

Figure 2 Mean + S.E.M. (A) GH, (B) lactate and (C) cortisol responses to two consecutive bouts of NMES administered at 2-h interval in six healthy subjects. Shaded areas represent the NMES exercise bouts and recovery. ${ }^{*}$ Significantly $(P<0.05)$ different than baseline values. ${ }^{\dagger}$ Significantly $(P<0.05)$ different than first bout values. 
significantly lower compared with the values measured at the same time during the first bout (Fig. 2C).

\section{Discussion}

It is well established that repeated GHRH stimuli (2-5) or voluntary exercise bouts $(6-8,15,16)$ administered at 2-h intervals are associated with a blunted $\mathrm{GH}$ responsiveness to the second stimulus in healthy adults, a restoration of $\mathrm{GH}$ responsiveness being present when the time elapsed between the two stimuli was more prolonged $(8,17)$.

We have recently demonstrated that a single bout of NMES exercise is able to induce markedly higher $\mathrm{GH}$ responses than those obtained with voluntary contractions of the same intensity (13). This finding is probably related to the higher degree of muscle fatigue, higher blood lactate concentration and pain induced by electrical muscle stimulation. Moreover, it cannot be ruled out that a higher core temperature induced by NMES (known to influence GH secretion) (18) might be involved in this pattern of $\mathrm{GH}$ responses.

The present study confirms that NMES administered at the same frequency $(75 \mathrm{~Hz})$ and intensity $(\sim 26 \%$ MVC force), but for a shorter period (9 min versus $18 \mathrm{~min}$ ) than in our previous study (13), is able to induce significant $\mathrm{GH}$ responses. These results indicate that $\mathrm{GH}$ responsiveness to NMES is strictly dependent on the duration of the stimulus. Indeed, mean peaks of GH were markedly higher in our previous study after $18 \mathrm{~min}$ of NMES $(12 \mathrm{ng} / \mathrm{ml})$ than those in the present study after only $9 \mathrm{~min}(4.5 \mathrm{ng} / \mathrm{ml})$.

It is of interest that the $\mathrm{GH}$ responses to two repeated bouts of NMES are persistent when the second stimulus was administered at a $2-\mathrm{h}$ interval. The persistence of $\mathrm{GH}$ responsiveness after the second bout of NMES resembles that observed with repeated pharmacological or voluntary exercise stimuli separated by a more prolonged period (4- and 6-h intervals) $(8,17)$.

Several mechanisms can be hypothesized to explain this different pattern of $\mathrm{GH}$ responsiveness occurring with NMES. The lower GH responses induced by the first NMES session (mean value: $4.5 \mathrm{ng} / \mathrm{ml}$ ) in comparison with those elicited by $1 \mu \mathrm{g}$ GHRH i.v. (mean value: $25 \mathrm{ng} / \mathrm{ml})(4,5)$ and/or voluntary exercise (mean value: $20 \mathrm{ng} / \mathrm{ml}$ ) (8) could be insufficient to deplete the immediately releasable $\mathrm{GH}$ pool and/or to cause a reactive increase in somatostatin, thus resulting in a similar response after $2 \mathrm{~h}$. Alternatively, it could be hypothesized that NMES circumvent standard feedback mechanisms through the involvement of other peripheral signalling pathways (i.e. stress-related mediators). In this respect, it is relevant to note the significant increase in the cortisol levels observed after the second bout of NMES, which could contribute in determining $\mathrm{GH}$ responsiveness.
The NMES-induced increase in blood lactate, which precedes the appearance of $\mathrm{GH}$ responsiveness by about 30 min after the two bouts, could also be involved in determining this pattern of GH responsiveness. Numerous studies have already reported a relationship between GH response and blood lactate accumulation (for review, (19)). Indeed, high concentrations of lactate within the muscle are reported to stimulate sympathetic nerve activity through a chemoreceptive reflex, which plays a role in the regulation of hypophyseal secretion of $\mathrm{GH}$ (19). However, the finding that NMES-induced lactate increase observed in the present study is markedly higher than that observed with the more prolonged duration of NMES in our previous experiments (in contrast to $\mathrm{GH}$ responsiveness) seems to rule out a central role of lactate changes (i.e. fatigue) in determining $\mathrm{GH}$ responsiveness to repeated NMES bouts.

Significant muscle fatigue occurred after both exercise bouts, as witnessed by the MVC force findings. Muscle fatigue could also be one of the factors explaining GH responsiveness after the different bouts, as proposed previously by Hakkinen \& Pakarinen (20). Surprisingly, the MVC force decrease was greater in this study after the two bouts of 20 NMES contractions $(\sim 37 \%)$ than after 40 consecutive NMES contractions $(\sim 22 \%)$ (13). However, the greater neuromuscular fatigue reported in the present study was not associated with a greater $\mathrm{GH}$ release. It is also interesting to remember that both in this and in our previous study, the subjects were stimulated at the maximal tolerated current level (pain threshold).

Even if muscle fatigue, blood lactate accumulation and pain may have played a role in the $\mathrm{GH}$ release during the two bouts, it seems plausible that the more potent stimulus to the NMES-induced GH responsiveness could be the duration of the exercise. In line with Stokes et al. (7), we conclude that the low GH responsiveness in the first bout, with $\mathrm{GH}$ returning to the initial value before the second bout, could allow $\mathrm{GH}$ release after $2 \mathrm{~h}$. Thus, repeated bouts of NMES exercise interspaced by $2 \mathrm{~h}$ can consistently stimulate $\mathrm{GH}$ secretion without attenuation of the GH response.

Further research is necessary to evaluate the changes in $\mathrm{GH}$ concentration after repeated bouts of voluntary isometric resistance exercise of the same force output in order to assess whether $\mathrm{GH}$ response is affected in the same manner after a voluntary protocol.

Although the persistence of $\mathrm{GH}$ responsiveness after repeated bouts of NMES could tentatively be useful to athletes for producing physiologically greater amounts of GH-insulin-like growth factor-I, the pain/discomfort associated with NMES, muscular damage and the high levels of fatigue make this approach impractical under the present experimental conditions. Further studies are necessary to verify other more acceptable and safe NMES modalities for increasing the endogenous GH production. 


\section{Acknowledgements}

The authors acknowledge Dr P G Marinone, Dr A De Col, Dr R Galli, Dr C Busti and Mr F Villano for their qualified assistance during the experiments and all the volunteers for their kind collaboration. We thank Dr S Lazzer for his contribution to the discussion of results and Dr J M H Buckler for the English revision. This study was partially supported by Progetti di Ricerca Corrente, Italian Institute for Auxology, Milano, Italy.

\section{References}

1 Müller EE, Locatelli V \& Cocchi D. Neuroendocrine control of growth hormone secretion. Physiological Reviews $1999 \quad \mathbf{7 9}$ 511-607.

2 Sartorio A, Spada A, Morabito F \& Faglia G. Different GH responsiveness to repeated GHRH administration in normal children and adults. Journal of Endocrinological Investigation 1988 11 727-729.

3 Ghigo E, Goffi S, Mazza E, Arvat E, Procopio M, Bellone J, Müller EE \& Camanni F. Repeated GH-releasing hormone administration unravels different $\mathrm{GH}$ secretory patterns in normal adults and children. Acta Endocrinologica 1989120 598-601.

4 Sartorio A, Spada A, Conti A, Grugni G, Morabito F \& Faglia G. Galanin infusion partially restores the blunted growth hormone responses to repeated growth hormone releasing hormone stimuli in normal adults. Journal of Endocrinological Investigation 199316 95-98.

5 Sartorio A, Conti A, Ferrero S, Spada A \& Faglia G. GH responsiveness to repeated GHRH or hexarelin administration in normal adults. Journal of Endocrinological Investigation 199518 718-722.

6 Cappon J, Brasel JA, Mohan S \& Cooper DM. Effect of brief exercise on circulating insulin-like growth factor I. Journal of Applied Physiology 199476 2490-2496.

7 Stokes KA, Nevill ME, Hall GM \& Lakomy HKA. Growth hormone responses to repeated maximal cycle ergometer at different pedaling rates. Journal of Applied Physiology 200292 602-608.

8 Sartorio A, Agosti F, Marinone PG, Proietti M \& Lafortuna CL. Growth hormone responses to repeated bouts of aerobic exercise with different recovery intervals in cyclists. Journal of Endocrinological Investigation $2005 \mathbf{2 8}$ RC11-RC14.
9 Lake DA. Neuromuscular electrical stimulation. An overview and its application in the treatment of sports injuries. Sports Medicine $199213320-336$.

10 Bax L, Staes F \& Verhagen A. Does neuromuscular electrical stimulation strengthen the quadriceps femoris? A systematic review of randomized controlled trials. Sports Medicine 200535 191-212.

11 McNeil CJ, Murray BJ \& Rice CL. Differential changes in muscle oxygenation between voluntary and stimulated isometric fatigue of human dorsiflexors. Journal of Applied Physiology $2006 \mathbf{1 0 0}$ 890-895.

12 Theurel J, Lepers R, Pardon L \& Maffiuletti NA. Differences in cardiorespiratory and neuromuscular responses between voluntary and stimulated contractions of the quadriceps femoris muscle. Respiratory Physiology and Neurobiology $2007157341-347$.

13 Jubeau M, Sartorio A, Marinone PG, Agosti F, Van Hoecke J, Nosaka K \& Maffiuletti N. Comparison between voluntary and stimulated contractions of the quadriceps femoris for growth hormone response and muscle damage. Journal of Applied Physiology (In press)

14 Sartorio A, De Nicolao G \& Liberati D. An improved computational method to assess pituitary responsiveness to secretagogue stimuli. European Journal of Endocrinology 2002147 323-332.

15 Cappa M, Bizzarri C, Martinez C, Porzio O, Giannone G, Turchetta A \& Calzolari A. Neuroregulation of growth hormone during exercise in children. International Journal of Sports Medicine 200021 (Suppl 2) S125-S128.

16 Stokes K. Growth hormone responses to sub-maximal and sprint exercise. Growth Hormone and IGF Research 200313 225-238.

17 Kanaley JA, Weltman JY, Veldhuis JD, Rogol AD, Hartman ML \& Weltman A. Human growth hormone response to repeated bouts of aerobic exercise. Journal of Applied Physiology 1997 83 1756-1761.

18 Christensen SE, Jorgensen OL, Moller N \& Orskov H. Characterization of growth hormone release in response to external heating. Comparison to exercise induced release. Acta Endocrinologica 1984 107 295-301.

19 Crewther B, Keogh J, Cronin J \& Cook C. Possible stimuli for strength and power adaptation: acute hormonal responses. Sports Medicine $200636215-238$.

20 Hakkinen K \& Pakarinen A. Acute hormonal responses to two different fatiguing heavy-resistance protocols in male athletes. Journal of Applied Physiology 1993 74 882-887.

Received 22 November 2007

Accepted 11 December 2007 